\title{
Research on Modern Costume Design Based on Computer Aided Design Means
}

\author{
Li Guo
}

Jiangxi Institute of Fashion Technology, Nanchang, 330000, China

Keywords: Computer aided design means, Modern Costume design, Research

\begin{abstract}
With the improvement of Chinese scientific and technological level, computer has gradually entered production and life with more and more obvious effect. It also plays an important role in the development process of clothing industry. Computer aided design first occurred in industrial design. However, it has gradually broken through the original design field and been applied to clothing industry widely with the progress and innovation of computer technology. The application of computer aided design in clothing industry starts from design phase. It has been gradually integrated into different links of clothing industry and then become an indispensable method in costume design, production and sales process.
\end{abstract}

\section{Introduction}

The coming of digital information era provides favorable development opportunities for costume design industry to a certain extent, especially the application of computer aided means in the process of costume design. Costume design is driven to a brand new development path through the full use of digital technology and visual performance language.

\section{Specific characteristics of computer aided design means in modern costume design}

\section{It has diversified expression means}

With corresponding reform and innovation during scientific and technological development, drawing tool has developed into digital hand drawing plate from the original lead pencil, thus promoting the progress and development of drawing skills. Brand new drawing tool can create brand new drawing means and then improve the pattern of manifestation of drawing effectively and expand the thinking and creative thought of designers. Computer graphics can be altered easily. Unsatisfying parts can be modified in time. Meanwhile, designers can master the shape, size and color comprehensively, thus avoiding the repeat of unnecessary work effectively. In costume design, designers need to describe contents such as costume outline, line, color and material texture with visual expression language and express simple, exaggerate or refined style of clothing vividly. In modern costume design, designers must master and understand computer drawing tool comprehensively. Computer aided design has many techniques of expression. The same software has different patterns of manifestation. Moreover, software categories are constantly increasing. Therefore, different software can be collocated and combined scientifically and rationally so as to complete costume design work better ${ }^{[1]}$.

\section{Computer aided design means runs through the whole process of costume design}

Computer design is available in each link of modern costume design, including costume making, printing, grading and style chart. Designers should choose computer software correctly in different stages of design so as to guarantee the quality of design work in each stage. Besides costume design phase, costume production process can also be controlled by computer and then the electronization of costume production can be realized. Work contents of multiple persons can be completed by the operation of only one person, which can save both time and manpower. In addition, computer aided design means can reflect the creative effect of costume design in virtual world so that designers can 
master actual operation accurately through virtual link. Meanwhile, corresponding brochures can be made by computer and clothing brand can be publicized through network. It is not difficult to publicize a brand through network due to the wider scope of network platform. It can master the initiative in publicity accurately, expand the coverage of information better and push clothing brand to the public.

\section{It has high integrity of data record and storage}

At the initial stage of formation of costume design concept, relevant plane graphs and videos can be collected on the network through computer and regarded as materials for creation; plane articles can be converted into digital documents that can be identified by computer through scanner and stored in the computer. If scanner cannot be used for scanning, digital camera can be used for photographing and then pictures can be saved in the computer. The pattern of computer storage is more convenient for the edition and design of stored data by designers and their application to costume design. Therefore, designers can collect, edit and combine image data through computer, save corresponding text ideas in design in the computer and modify and view them at any time. When completing a design scheme, designers can save effect picture, paper pattern, process and data in the computer or in other memorizers through computer and encode and file them. When meeting the same problem in later period, they can provide effective data and then reduce the time of repeated work. Such storage means has a longer storage time than paper. Meanwhile, it is safer and can save real space.

\section{It is good for better communication with customers}

In the whole process of costume design, designers can make network contact with customers through computer, then deliver design works and production process problems to customers in the first time and guarantee timely solving of corresponding problems. Such communication form can save the time of designers effectively, reduce transportation expenses of matter transmission, expand customer groups and break through limitations of local customer groups. It is an advanced marketing approach currently. In addition, when showing relevant design scheme to customers, designers can make explanations through computer and use output tools externally connected to computer in combination with image and sound effect so as to present costume design concept and effect perfectly and create a high-quality environment of appreciation for customers. Meanwhile, the design effect can be presented better with the aid of computer ${ }^{[2]}$. Under the situation of computer aid, designers can make improvement and adjustment according to the specific situation of project design and modify the content and pattern of manifestation based on specific demands.

\section{Realization of modern costume design concept by computer aided design means}

\section{Manifestation of garment fabric by computer aided design means}

The most important carrier of expression of the overall garment effect is fabric which occupies the main position in clothing area. The pattern and texture of garment fabric and secondary new texture directly manifest the personality of designers and the spirit of the work. Therefore, the selection of garment fabric is very important in costume design and completion process. In reality, fabric pattern and texture provide design inspiration for designers. Meanwhile, the design idea and draft of designers can provide a correct direction for fabric selection. Therefore, they supplement and promote each other and jointly develop. Important constituent parts of fabric are pattern and texture which cannot exist independently. The same pattern with different textures will produce different visual effects. The same texture of different patterns has different effects of expression. Therefore, costume designers should attach importance to the design of garment fabric.

Costume designers need to choose appropriate fabric type, pattern and color correctly according to the design concept and the current fashion trend. However, the selection of fabric should better be completed through computer. Designers can convert physical fabric into digital documents that can 
be identified by computer through a scanner or camera, choose appropriate fabrics with software based on actual demands or design the required fabric with computer and then choose the optimal fabric in the shortest time ${ }^{[3]}$. In the process of seeking for fabric, if the fabric required by designers is not available in the market, it can be bought on the internet where there are varieties of fabrics. Therefore, more fabrics can be found through network platform which can break through geographic restrictions and expand the business scope of fabric suppliers. In addition, fabrics made with computer software can be made with machine controlled by computer or by hand. Therefore, computer aided technology is very important for modern costume designers.

Texture performance of fabric

Model evaluation is mostly determined by touching experience in the life of modern people. Due to various varieties and different textures of fabrics, their appropriate use will produce different styles and sense of beauty. Worsted makes people warmer, chiffon makes people feel light-footed and romantic, knitted fabric looks more casual and rough linen gives people a feeling of returning to their original nature. In essence, texture is mainly manifested by chemical and physical properties of materials. Designers' mastery of fabric is similar to people's mastery of body language. With appropriate mastery of body language, the interior emotion of people can be presented fully. With appropriate mastery of fabric, the interior emotion of costume design can be reflected. People have gradually turned to artistic value from practical value of clothing in the constant development process of the society. Therefore, single fabric can no longer meet the demand of people for artistic value of clothing. It is necessary to make full use of computer aided design means to design more garment fabrics and simulate the effect of finished fabrics practically under the current situation of scientific and technological development.

Though texture performance of fabrics cannot express all characteristics of fabrics completely, special features can be extracted. Meanwhile, texture performance of fabrics has direct influence on the selection of garment fabric. Though hand drawing can show the texture of fabrics, its form is too single. Fabric texture can be shown with multiple methods by computer aided means. Filter gallery in computer Photoshop software has many effects of expression. Data can be adjusted according to actual demand. Different combinations of filter gallery can show different effects and make texture more real. This cannot be achieved by hand drawing ${ }^{[4]}$.

Pattern design of garment fabric

Pattern design of garment fabric plays an important role in the process of modern costume design. Patterns mainly include single pattern and continuous pattern. With certain independence, single pattern can be designed according to the overall outline of garment or independently. Its structure and shape are complete. It can be designed based on the creative thinking of costume designers. Continuous pattern refers to the combination of basic unit patterns in a rhythmic and regular way which makes pattern arrangement more rhythmic and stratified. However, computer has strong graphic processing ability. It can aid in completing the design work of garment fabric and achieve digital sample making. Computer Photoshop software can process pictures and images. Though it is a two-dimensional graphic design software, it can present three-dimensional effect. In addition, CAD software can design a screen as paper. This system can make arrangements for yarns as specified by designers, simulate yarn texture image automatically and finally form a simulation image of fabric with a visual sense consistent with the reality.

Plane design also plays a vital role in the design process of garment fabric. Both single pattern and continuous pattern must be designed according to composition rules of plane design. In the current stage, the effect of fabric design has gradually developed towards three-dimensional and multilevel direction. Ideal simulation effect can be produced with different tools of computer software. For check fabric design, line tool in the computer software can produce vertical and crossed lines which can form sparse, dense and orderly checks. Then, checks with dark color can be subject to space opening processing, thus forming a vivid three-dimensional space. 


\section{Effect picture and style chart of costume design}

Fashionable dress refers to clothing accepted by most people in the specific time and region. Fashionable dress has strong periodicity. Due to the constant improvement of social cultural and economic level, its fashion cycle has shortened constantly. Fashionable dress has a great price difference in different stages of the same period. Therefore, to guarantee long-term existence of a fashionable dress brand, it is necessary to adjust products with the change of era, but the essential concept remains unchanged. As fashionable dress has strong particularity of times which is mainly manifested in fashion style, wearing way and color, the particularity of times must be manifested when garment effect picture is drawn.

Effect picture of fashionable dress should be first drawn by hand and then delivered to the computer through tools such as camera or scanner. Then, it is necessary to modify the picture and adjust its color in the computer. The required background or accessory can be added. Then, it should be printed with digital printer. In addition, effect picture can be drawn by computer and relevant vector diagram software can be used to draw fashion style and outline. Then, the finished outline should be delivered to PS software and subject to color filling through color filling tool. The designed fabric can be filled into garment outline. Then, different effect tools in the software can be used for synthesis so as to achieve the design effect of designers. After the completion of design of garment effect, garment collocation and accessories and the wearing environment can be integrated into the final effect picture.

\section{Conclusion}

In conclusion, computer aided means plays an important role in modern costume design. It is also the main power of development of clothing industry. Computer aided design means is an advanced technology. In costume design process, computer aided design means is not simply an independent artistic form. It does not have independent ornamental value and is different from traditional design concept. It needs to follow computer technology. Moreover, successful design works should not only have certain expressive force of design, but also reflect the design personality of designers. On this basis, modern costume designers should have certain design accomplishment and creative ability, master creation concept and inspiration accurately and reflect creative characteristics actually. Computer aided design means plays an important role in modern costume design. It has broken through deficiencies and shortages of hand drawing, innovated the method and approach of modern costume design and promoted the development of modern costume design industry.

\section{References}

[1] Jiang Meizhen, Ouyang Yan'e. New Concept of Modern Costume Design Networking - Virtual Costume Design. China Science \& Technology Panorama Magazine, 2010(10):183.

[2] Fang Linlin, Yu Meilian. Study on Application of Computer Embroidery in Modern Chinese Costume Design. Popular Literature, 2015(8):61-62.

[3] Huang Wei. Analysis on Influence of Computer Science and Technology on Students Majoring in Clothing. Light Textile Industry and Technology, 2014(6):139-140.

[4] Ding Chengyan. Embodiment of Chinese Elements in Modern Costume Design. Value Engineering, 2013(25):280-281.

[5] Li Yi, Wei Linlin. Analysis on Artistic Expression of Quilting Seam in Modern Costume Design. Wool Textile Journal, 2015,43(6):61-64. 